\title{
Perspective
}

PERSPECTIVE Actualité en histoire de l'art

$2 \mid 2007$

La Grande-Bretagne/Période moderne

\section{L'architecture gothique dans les îles Britanniques : orientations et perspectives de la recherche}

Gothic architecture in Britain. Some observations on current writing and research

Die gotische Architektur in Groß-Britannien: einige Bemerkungen zum aktuellen Stand der Literatur und Forschung

Architettura gotica in Gran Bretagna: alcune osservazioni sugli studi e sulle ricerche in corso

Arquitectura gótica en Gran Bretaña: algunas observaciones sobre la literatura y las investigaciones actuales

\section{Roger Stalley}

\section{(2) OpenEdition}

Journals

Édition électronique

URL : http://journals.openedition.org/perspective/3812

DOI : 10.4000/perspective.3812

ISSN : 2269-7721

Éditeur

Institut national d'histoire de l'art

Édition imprimée

Date de publication : 30 juin 2007

Pagination : 261-280

ISSN : 1777-7852

\section{Référence électronique}

Roger Stalley, "L'architecture gothique dans les îles Britanniques : orientations et perspectives de la recherche », Perspective [En ligne], 2 | 2007, mis en ligne le 31 mars 2018, consulté le 01 octobre 2020. URL : http://journals.openedition.org/perspective/3812 ; DOI : https://doi.org/10.4000/perspective. 3812

Ce document a été généré automatiquement le 1 octobre 2020. 


\title{
L'architecture gothique dans les îles Britanniques : orientations et perspectives de la recherche
}

\author{
Gothic architecture in Britain. Some observations on current writing and \\ research \\ Die gotische Architektur in Groß-Britannien: einige Bemerkungen zum aktuellen \\ Stand der Literatur und Forschung \\ Architettura gotica in Gran Bretagna: alcune osservazioni sugli studi e sulle \\ ricerche in corso \\ Arquitectura gótica en Gran Bretaña: algunas observaciones sobre la literatura y \\ las investigaciones actuales
}

Roger Stalley

\section{NOTE DE L'AUTEUR}

Cet article est la version augmentée d'une conférence donnée au Yale Center for British Art en novembre 2006 et consacrée à l'avenir de l'histoire de l'architecture britannique. Je souhaite remercier Frank Salmon, qui m'a sollicité pour cette intervention, et Peter Draper qui a présidé la session dans laquelle elle a eu lieu. J'ai eu l'occasion de discuter depuis des années des questions soulevées par cet article avec de nombreux collègues et amis, dont Lawrence Hoey à la mémoire duquel j'aimerais rendre hommage. Pour leurs précieux conseils pour la mise en forme finale du texte je suis très reconnaissant à Edward McParland et Danielle O'Donovan. 


\section{Une discipline en crise, un objet ouvert ?}

\section{La nécessité d'une nouvelle synthèse}

1 Il y a une quarantaine d'années, les études sur l'architecture médiévale présentaient en Grande-Bretagne un tableau relativement clair. Elles étaient dominées par Nikolaus Pevsner, alors plongé dans l'écriture des volumes de la collection «Buildings of England ", un travail réputé pour la concision de ses commentaires et la netteté de ses classifications. Il a fallu attendre les années 1970 pour que ses convictions historicistes soient remises en cause, et notamment l'idée que les édifices anciens participent d'une histoire continue dont le cours se déroulerait inexorablement jusqu'à nous (WATKIN, 1977) ${ }^{1}$. Les postulats de N. Pevsner nous paraissent moins assurés à présent, confrontés que nous sommes à une grande variété de points de vue, allant de l'analyse formelle attributionniste façon Morelli au discours postmoderniste. Mais quelles que soient les limites de sa démarche, on ne saurait ignorer son retentissement sur les études d'architecture anglaise (non pas britannique, cependant); virtuellement, tous les édifices gothiques de quelque importance sont concernés par son approche et, de ce fait, il est difficile d'échapper à son influence ${ }^{2}$.

2 Parmi ses contemporains, N. Pevsner demeure sans rival, eu égard à l'ampleur du travail accompli et à l'envergure de sa vision. De fait, la seule synthèse moderne dont nous disposions pour l'architecture médiévale britannique a été écrite dans une perspective économique et sociale plutôt que proprement architecturale (PLATT, 1990), et elle n'a guère reçu un accueil favorable de la part des historiens de l'architecture. La dernière étude substantielle en ce domaine remonte donc à un demi-siècle : il s'agit du volume de Geoffrey Webb pour la collection «Pelican History of Art » dont Pevsner était d'ailleurs l'éditeur (พЕEBB, [1956] 1965)³. Cette situation n'a rien de surprenant, étant donné la diversité et le nombre des publications qui ont paru dans l'intervalle. Cependant, ainsi que le constatait Geoffrey Webb, " pour chaque génération, la même nécessité s'impose non seulement de réviser, à la lumière des travaux accumulés, nos propres points de vue sur un sujet si considérable (celui des édifices médiévaux), mais encore de récrire son histoire à la lumière des préoccupations de la génération nouvelle » (WEBB, 1965, p. xxi) ${ }^{4}$ - et ce bien qu'une étude d'ensemble fasse défaut et que nous n'ayons rien en tout cas qui puisse être comparé à celle de Norbert Nussbaum sur les églises allemandes (nussbaum, 1994; nussbaum, 2000), même si l'ouvrage essentiel de Peter Draper sur le premier gothique anglais a réussi à placer l'étude de l'architecture ecclésiastique des années 1170-1250 à un niveau inédit et remarquable (DRAPER, 2006). Un effort de synthèse est toujours nécessaire, ne serait-ce que pour proposer des problématiques nouvelles et esquisser les cadres d'analyse possibles de futurs travaux. Actuellement, qui chercherait une introduction générale au domaine anglais aurait intérêt à consulter, plutôt qu'un seul ouvrage, les brefs essais publiés dans les catalogues des deux expositions londoniennes The Age of Chivalry (Age of Chivalry, 1987) et Gothic Art for England (Gothic Art for England, 2003). Pour le gothique tardif, le résumé de Christopher Wilson dans le dernier de ces catalogues est particulièrement utile ${ }^{5}$. 


\section{Une histoire de l'architecture, ou une histoire matérielle/culturelle ?}

3 N. Pevsner et G. Webb étaient, l'un comme l'autre, historiens d'art. Or au sein de l'université, les historiens de l'architecture médiévale ont tout l'air aujourd'hui d'une espèce menacée. Bien plus, l'approche traditionnelle en ce domaine a subi les critiques de représentants d'autres disciplines ${ }^{6}$. Un excès de formalisme, une analyse trop exclusivement attachée aux étapes de la construction, tels sont les reproches les plus courants. Un commentateur, archéologue de formation, parle «d'immobilisme méthodologique » et appelle de ses vœux une recherche qui saurait intégrer les édifices dans le contexte de l'histoire politique et culturelle (o'KEEFE, 2006, p. 258-62) ${ }^{7}$. Bien évidemment, ce genre de point de vue a pour conséquence logique d'englober l'histoire de l'architecture dans un discours historique plus large, les édifices n'étant plus envisagés en eux-mêmes mais comme des expressions de la sensibilité religieuse, de la structure sociale, de la répartition des richesses ou de l'idéologie politique. Le principe n'a rien d'irrecevable, sauf s'il se concrétise aux dépens de l'analyse architecturale. Mais voici un second grief : cette matière que les historiens de l'architecture savent si bien réunir et inventorier, ils seraient parfois incapables de l'assimiler et de lui donner un sens. Une récente publication consacrée aux maisons médiévales anglaises, corpus utile de l'habitat encore existant, s'est attiré un commentaire acerbe et le reproche d'avoir "obscurci», par souci du détail architectural, "les motifs essentiels du développement de la demeure médiévale: distinction sociale, renforcement de la sphère intime, raffinement accru des modes de vie » (EMERY, 2005 ; GOMME, 2006, p. 18) . $^{8}$

Dans le même temps, l'historien d'art est serré de près par des concurrents issus d'autres disciplines et désireux d'étendre leur domaine. Pour certains chercheurs postmodernistes, l'architecture n'est tout au plus qu'une facette de l'histoire de la culture et des idées; Paul Binski est le meilleur représentant de cette approche en Angleterre (BINSKI, 1995 ; BINSKI, 2005); d'autres, archéologues de profession, se sont emparés de l'aspect technologique de la question. Aux yeux des historiens traditionalistes, les archéologues ont « accaparé » l'architecture médiévale, à force de répéter que cette discipline ne représente ni plus ni moins qu'une "archéologie verticale». Eric Fernie a proposé une mise au point judicieuse sur les rapports de l'histoire de l'art et de l'archéologie (FERNIE, 2003). Une autre critique, souvent reprise, concerne l'étroitesse excessive des études architecturales. Il est vrai que la formation des historiens de l'art les incite à se limiter aux grandes cathédrales et aux monastères. Les églises paroissiales sont abandonnées, pour une grande part, aux historiens et érudits locaux ${ }^{9}$, les châteaux sont l'apanage des archéologues $^{10}$ et les édifices vernaculaires ont leurs propres spécialistes. Cela est dû, bien sûr, au fait que les historiens de l'art, privilégiant les qualités formelles, se concentrent nécessairement sur les réalisations les plus accomplies d'une époque. Une nouvelle famille d'auteurs, venus de l'archéologie, se distingue par l'ardeur de sa prédication: elle s'en prend volontiers aux «tenants de la tradition » et congédie dans certains cas la discipline dans son ensemble comme «non scientifique » ou d'esprit « antiquaire» (quand elle ne lui reproche pas de combiner ces deux défauts). Trop souvent, ces attaques s'adressent à un adversaire inexistant ou qui du moins a disparu depuis plusieurs décennies, comme l'a brillamment montré Richard Morris (dans le compte rendu d'un ouvrage consacré à l'architecture castrale ; MORRIS, 2003). 
5 Les historiens de l'architecture se trouvent ainsi confrontés à un défi de première importance : dans quelle mesure doivent-ils se concentrer sur l'étude du style, et dans quelle mesure sur l'étude des bâtiments? S'agit-il principalement de comprendre le gothique dans ses diverses expressions ou bien d'étendre l'analyse à tous les monuments, quels que soient leur type ou leur forme et sans égard pour leurs qualités formelles? Ceux qui leur reprochent un intérêt exclusif pour la plastique des édifices et pour les monuments d'«élite» font d'ailleurs écho à une controverse interne: l'histoire de l'art doit-elle s'étendre à toutes les manifestations de la culture visuelle ou se restreindre aux œuvres dont la valeur esthétique est la plus remarquable (BREDEKAMP, 2003, entre autres) ? Michael Davis a récemment formulé le problème, en signalant les dangers d'une « enquête exclusivement fondée sur la base de ce que Christophe Wilson a appelé 'the great church' et qui considérerait les réalisations d'une extravagance ou d'une complexité manifestes comme les seules œuvres réellement significatives " (DAVIS, 2001, p. 93). Poussée à l'extrême, l'approche « élitiste » a pour effet d'exclure de notre champ la plupart des églises paroissiales, sans parler des châteaux et autres édifices profanes ${ }^{11}$. Une conséquence qui se fait notamment sentir dans le cas de l'architecture monastique, où l'on s'est trop concentré sur les églises, au détriment, souvent, des autres bâtiments qui dépendaient du monastère. Signe heureux de changement, la livraison 2006 du British Archaeological Journal était entièrement consacrée au dessin des cloîtres (HENIG, MACNEILL, 2006). Nancy Steiber a clairement résumé les récents changements d'approche dans les études architecturales: «Nous nous sommes éloignés des thèmes qui caractérisaient précédemment l'histoire de l'architecture: présentation héroïque des architectes, célébrations de l'héritage national, prises de position dans les controverses architecturales de l'heure ou sujets qui venaient renforcer le point de vue canonique »(STEIBER, 2003, p. 176) ${ }^{12}$.

\section{Classifications stylistiques et schéma d'évolutions}

6 L'un des aspects les plus intéressants du livre publié en 1956 par Geoffrey Webb est sa tentative d'échapper aux classifications stylistiques qu'avait conçues Thomas Rickman au XIX siècle: premier style anglais (early english), style orné (decorated) et style perpendiculaire (perpendicular). On sait depuis longtemps combien ces catégories sont inadéquates, mais il est difficile de remettre en cause leur prégnance. L'essai de T. Rickman s'adressait aux architectes de métier plutôt qu'aux historiens et, ainsi que le déplorait Francis Bond voilà un siècle, ses catégories ne s'appliquent en toute rigueur qu'au tracé des fenêtres (BOND, 1906, p.xix-Xx) ${ }^{13}$. Ces classifications traditionnelles trouvent leur origine dans l'architecture ecclésiastique (c'est aussi le cas de la terminologie française) et s'adaptent mal aux bâtiments séculiers : cette difficulté met en exergue ce qui distingue les deux domaines de notre discipline. Ainsi, les châteaux gallois du temps d'Edouard $\mathrm{I}^{\mathrm{er}}$ passent pour des produits de la période dite du style orné (decorated style), terme qui ne s'impose pas à l'esprit lorsque l'on considère l'imposante maçonnerie des châteaux de Conway ou d'Harlech ${ }^{14}$. Il faut aussi souligner que les classifications de T. Rickman sont peu pertinentes hors des frontières de l'Angleterre en Écosse, au Pays de Galles, en Irlande - où elles ont entravé plutôt que favorisé l'analyse.

7 Si les catégories traditionnelles appartiennent au passé, quels sont les autres cadres d'analyses applicables au gothique anglais ou, pour mieux dire, britannique? 
Manifestement, les schémas linéaires qui sous-tendaient l'étude du gothique français sont tout aussi dépassés. Comme l'observait Willibald Sauerländer, « la plupart de nos travaux concernant la première architecture gothique furent dominés par la conviction positiviste qu'une chronologie linéaire pouvait rendre compte sans ambiguïté de toutes ses caractéristiques, de tous ses signes distinctifs " (SAUERLÄNDER, 1995, p. 6) ${ }^{15}$. On connaît les risques inhérents à ce genre d'approche, et en particulier le fait qu'en pareil cas, les similitudes se trouvant inévitablement valorisées aux dépens des différences, les auteurs auront tendance à négliger les œuvres atypiques, notamment celles qui ne s'intègrent pas dans un processus "d'évolution ». La question des étapes initiales du gothique anglais illustre bien le problème, tant les chercheurs ont eu de mal à dresser un tableau cohérent de cette période : aucun centre n'est identifiable à partir duquel les conceptions gothiques se seraient diffusées vers une éventuelle périphérie ; les chronologies se chevauchent; les principaux monuments ont disparu. Aussi est-il difficile, sinon impossible, de dégager les lignes directrices d'un quelconque développement (à supposer qu'elles aient jamais existé). Nulle exigence de conformité ne s'imposait alors: on a pu le constater dans l'Ouest, où les bâtisseurs d'édifices voisins, comme la cathédrale de Wells et l'abbaye de Glastonbury, étaient loin d'entendre le gothique dans les mêmes termes. Au même moment, dans le sud du Pays de Galles, les maîtres d'œuvre de Saint-David édifiaient une église qui présente tous les caractères $d u$ "roman flamboyant». Ces trois monuments étaient en cours de construction dans les mêmes années 1180-1190. Le terme "de transition » n'est pas moins trompeur, qui laisse entendre que l'architecture anglaise allait évoluant vers un modèle préexistant. En réalité, la période de formation du gothique anglais fut une époque éminemment inventive et expérimentale. Face à une telle complexité, il faut bien convenir, comme l'écrivait naguère Paul Crossley, que «les édifices sont des réponses particulières à des problèmes particuliers et non les éléments coordonnés d'un processus historique harmonieux» (CROssLey, 2001, p. 95). C'est le point de vue qu'a adopté Paul Draper dans un livre récent, où toute notion de progression narrative est judicieusement écartée (DRAPER, 2006). Il se compose de chapitres traitant successivement de la nature de la relation de commande, de l'église paroissiale, de l'identité nationale et des relations de la liturgie et de l'architecture. L'un des thèmes les plus intéressants est celui du régionalisme, avec ce problème qu'en l'absence d'un grand centre ou d'un courant dominant en Grande-Bretagne, il est difficile de décider ce qui peut-être qualifié ou non de « régional ».

\section{Questions en cours}

\section{Le premier gothique : le jeu des influences françaises}

8 Les difficultés que présente l'architecture anglaise se font particulièrement sensibles pour les premières étapes du gothique, surtout lorsqu'on entre dans le détail de cette période. Comment les idées françaises sont-elles entrées en contact avec les ateliers anglais? Jusqu'à quel point les commanditaires anglais connaissaient-ils les édifices français? Le gothique ne s'est pas imposé comme un style complet et intégral : il semble que l'adoption des techniques françaises ait dépendu des circonstances plutôt que d'un ordre préétabli. Même la présence d'un maitre maçon français à Canterbury ne parvint pas à produire une structure nettement française de caractère : en faisant appel à Guillaume de Sens, les moines choisissaient un homme plutôt qu'un style. Les 
racines françaises de Canterbury furent mises en évidence voilà plus d'un demi-siècle par Jean Bony (BONY, 1949) et si son analyse a pu être précisée depuis, elle demeure inchangée pour l'essentiel (GRANT, 1991, p. 117 et n. 19; MAIR, 1982) ${ }^{16}$. Ces dernières années, d'autres thèmes ont retenu l'attention, notamment les étapes de la construction, la configuration initiale du sanctuaire et de la "corona» [la chapelle axiale] et les intrigues ecclésiastiques auxquelles la reconstruction a donné lieu. Pour toutes ces questions, des interprétations sensiblement divergentes ont été proposées tour à tour par Peter Draper, Millard Hearn et Peter Kidson, ce dernier avançant que le sinistre de 1174 qui détruisit le chœur roman pourrait bien être un incendie volontaire (DRAPER, 1983 ; DRAPER, 2006 ; HEARN, 1994 ; KIDSON, 1993). Il y a peu, Lawrence Hoey a comparé ces différents points de vue dans un article particulièrement suggestif (HOEY, 1995). Le grand avantage, dans le cas de Canterbury, est bien sûr que Gervase nous a laissé le récit de sa reconstruction, un texte qui a permis d'illustrer les processus de construction à travers toute l'Europe. Cependant, les mots qu'il emploie méritent qu'on les déconstruise avec soin, ainsi que l'a souligné Carol Davidson Cragoe (DAvidson CRAGOE, 2001). Bien qu'on ait décelé l'influence de Canterbury dans des réalisations plus tardives, comme les chœurs des cathédrales de Lincoln et de Chichester, personne n'a $\mathrm{pu}$ mettre en évidence son retentissement sur l'architecture anglaise dans son ensemble. On estime à présent que le second architecte de Canterbury, Guillaume l'Anglais, fut associé à des constructions plus lointaines, celle en particulier de la cathédrale de Lausanne ${ }^{17}$. Bien que l'influence de Canterbury soit incontestable, on ne saurait en faire la «source» et "l'origine» du gothique anglais, même si une évaluation d'ensemble de l'influence de Canterbury sur l'architecture anglaise serait nécessaire. Christopher Wilson a mis en lumière des éléments plus précoces dans la Temple Church de Londres, datant sa rotonde des années 1160, pour conclure qu'elle " présente toutes les caractéristiques d'une conception due à un architecte de Picardie ou des Flandres françaises » (WILsON, 1986, p. 91, n. 14) ${ }^{18}$; mais l'édifice est consacré en 1185, et la datation précoce de 1160 a été mise en doute. Les idées françaises furent également exploitées dans de nombreux édifices de la côte Sud (GRANT, 1991; GRANT, 1994), comme New Shoreham et Boxgrove, bien qu'aucun d'entre eux ne se puisse confondre avec les monuments français.

9 La situation est tout aussi complexe dans l'Ouest de la Grande-Bretagne. Ici non plus, aucun monument particulier qui fasse office de modèle, bien qu'on retrouve des caractères communs, identifiés depuis longtemps par Brakspear dans son célèbre article sur "the West Country School», avec pour édifices majeurs la cathédrale de Worcester (les baies occidentales, sur lesquelles voir également wILSON, 1975), l'église cistercienne de l'abbaye de Dore, la cathédrale de Wells, l'abbaye de Glastonbury et la cathédrale Saint-David (BRAKSPEAR, 1931). Carolyn Malone a étudié en détail les qualités formelles de ces bâtiments dans sa thèse (non publiée) de 1973 (MALONE, 1973). Pour la grande église de Glastonbury, une étude moderne manque ; en revanche, des articles ont récemment été consacrés à la cathédrale Saint-David (DRAPER, 1999; STALLEY, 2002) et à l'abbaye de Dore (MALONE, 1984 ; O'CALLAGHAN, 1995 ; THURLBY, HARRISON, 1997). Malgré ces travaux, rien ne permet d'établir avec certitude les modalités de diffusion du gothique dans l'Ouest de l'Angleterre. Nous ne sommes plus au temps où John Harvey pouvait présenter la cathédrale de Wells comme le premier exemple de l'architecture gothique (non seulement d'Angleterre, mais d'Europe). De fait, la genèse précise du dessin de la cathédrale de Wells demeure une énigme, que Caroline Malone a 
récemment envisagée dans le contexte de l'art cistercien (MALONE, 1984 ; MALONE, 2004a ; MALONE 2004b).

Le premier gothique du nord de l'Angleterre a suscité une véritable avalanche de publications, et c'est sur ce thème que les débats furent les plus passionnés. Ils concernaient, pour la plupart, le rôle des cisterciens et le degré de responsabilité de l'ordre quant à l'introduction du gothique dans cette région. Divers témoignages ont été invoqués. Peter Fergusson a souligné les caractéristiques françaises de l'église cistercienne de Roche, les rattachant à des sources du nord-est de la France; en outre, il a désigné l'abbaye de Byland comme un monument majeur (FERGUSSON, 1971; FERGUSSON, 1975). M. Hearn a mis en avant l'exemple de Ripon Minster, pour soutenir que son dessin dérivait de la cathédrale de Laon (HEARN, 1983; pour une critique de ce point de vue, voir HARRISON, BARKER, 1999). Dans le même temps, C. Wilson signalait l'importance de l'abbaye de Furness (wILSON, 1986) et Malcolm Thurlby mettait en lumière les éléments les plus précoces de la cathédrale Saint-Andrews (THURLBY, 1994). Dans toutes ces discussions, il était beaucoup question des «prototypes perdus » du nord de la France, quoiqu'en bien des cas on fût loin de pouvoir les situer ou les identifier avec précision. Néanmoins, on s'accorde généralement à reconnaître l'importance capitale du chœur de York Minster, réalisé du temps de l'archevêque Roger Pont l'Evêque (1154-1181). Les parties de la crypte qui subsistent suggèrent une robuste empreinte du roman tardif, mais les moulurations retrouvées du corps principal de l'édifice donnent l'impression d'une structure plus légère et plus élégante, hypothèse dont M. Thurlby a mesuré la probabilité (THURLBY, 2000). Quoique nous connaissions mieux à présent les édifices du Nord, le rôle des cisterciens demeure difficile à cerner car les églises sont en ruine et leurs chronologies peu précises. Les découvertes les plus stimulantes proviennent de l'analyse des éléments de maçonnerie rapportés, et notamment de la patiente enquête menée par Stuart Harrison qui a permis de restituer les traits principaux des plans d'origine: ceux, entre autres, de l'élévation de la nef à Byland et le tracé de la fenêtre de la façade occidentale (HARRIsON, BARKER, 1987 ; HARRISON, 2003). Parmi les nombreux apports de ce travail, nous mesurons mieux désormais l'importance de la rosace dans l'architecture gothique du Nord.

\section{Identité nationale et perception du gothique anglais}

Tandis que les spécialistes s'employaient à débrouiller cet écheveau stylistique, ils accordaient moins d'attention à un autre problème: pourquoi la physionomie des édifices anglais et français diffère-t-elle à ce point? Est-ce une question de choix personnel, la marque d'une divergence dans les valeurs esthétiques adoptées de part et d'autre ou le signe d'un nationalisme naissant? Le gothique anglais a privilégié davantage ce qu'on peut appeler les éléments décoratifs, obéissant en certains cas à des considérations idéologiques, ce que $\mathrm{P}$. Binski a démontré de manière convaincante dans ses deux ouvrages récents (BINSKI, 1995; BINSKI, 2005). Mais le facteur le plus déterminant fut sans doute l'échelle des édifices, héritage de la période romane. La plupart des cathédrales, ainsi que les principales églises bénédictines, ont été reconstruites en grand nombre durant l'ère normande, et les constructions gothiques ont généralement pris la forme d'extensions ou d'éléments de raccord. Comme l'a remarqué Lawrence Hoey, le roman anglo-normand était si monumental, si imposant, qu'on pouvait difficilement le remplacer ${ }^{19}$. En conséquence, la structure des églises 
anglaises présente un dispositif moins rigoureusement ajusté qu'il ne le fut en France. Aussi a-t-on pu parler d'un gothique « de surface » (cosmetic Gothic), ce qui signifie que les éléments gothiques furent seulement greffés sur des structures traditionnelles. Mais les choses ne sont pas si simples. Les bâtisseurs anglais ont inventé un gothique autre, riche en surprises visuelles et en allusions spirituelles, comme l'a montré P. Binski. Il nous faudrait en savoir davantage sur les commanditaires, sur la manière dont ils percevaient les œuvres qu'ils finançaient. Une source longtemps négligée, la Vie de saint Hughes, a été récemment étudiée par Alexander Heslop (HESLOP, 2000). Ce poème composé vers 1230 relate la vie de l'évêque Hugues d'Avalon, fondateur de la cathédrale gothique de Lincoln quelque quarante ans auparavant, et contient, parmi d'autres allusions à la beauté de l'édifice, un extraordinaire commentaire sur la voûte à nervures, laquelle " étend largement ses vastes ailes tel un oiseau ailé en plein vol ", «atteint les nuages, tout en se maintenant sur ses solides colonnes ${ }^{20}$ (LEHMANBROCKHAUS, 1955, n² 2372).

12 Le problème de la perception du gothique a intéressé Lawrence Hoey : il s'est penché sur l'une des énigmes de l'architecture gothique anglaise, l'extraordinaire variété des formes qu'affectent les piliers. Il montre de façon convaincante que cette question complexe est au cœur du sujet (HOEY, 1986 ; HOEY, 1987 ; HOEY, 1994). Dans bien des cas, cette diversité ne correspond à aucun système d'alternance identifiable, et il semble que l'inventivité l'emporte sur la cohérence. La nef de Selby est un exemple notoire de ce triomphe du caprice (stalley, 1995) ${ }^{21}$. Dans la pratique, les perceptions de la beauté architecturale s'éloignaient nettement des théories en honneur dans la philosophie scolastique, les chroniqueurs préférant des épithètes telles que incomparabilis ou sumptuosus aux notions d'harmonie et de concordance héritées de Vitruve ${ }^{22}$. Cette question de la perception ramène à la problématique d'une identité nationale en architecture (qui avait fait l'objet d'utiles remarques de P. Binski ; BINSKI, 1995) et vient d'être abordée dans le récent ouvrage de P. Draper (DRAPER, 2006). Il s'interroge en particulier sur l'homogénéité croissante des édifices anglais après 1220 et sur les raisons qui les firent différer à ce point de l'architecture française. L'ignorance n'est pas en cause, puisque les membres du clergé insulaire eurent maintes occasions d'étudier les constructions françaises durant les années d'exil, au temps de l'Interdit (1208-1213 $)^{23}$, sous le règne du roi Jean. Après leur retour du continent, on aurait pu s'attendre au contraire à un regain de l'influence française. Pour expliquer ce constat, $P$. Draper repère diverses manifestations de nationalisme dans plusieurs domaines de la vie publique, et en particulier dans le culte des saints indigènes. Cependant, les textes contemporains faisant défaut, on ignore si les commanditaires anglais et les maîtres maçons ont cultivé l'admiration de l'art français ou s'ils ont consciemment tiré fierté du caractère anglais de leurs propres œuvres.

\section{L'importance d'une approche globale}

13 On a tendance à négliger ces questions de grande ampleur dans un domaine où les études monographiques sont devenues majoritaires. Ainsi une recension des articles publiés sur une période de dix ans (1995-2005) par le Journal of the British Archaeological Association (la principale revue d'architecture ecclésiastique médiévale) révèle que sur vingt-sept articles, dix-sept sont consacrés à un édifice particulier. De même la plupart des conférences annuelles de la British Archaeological Association sont consacrées à 
des édifices particuliers (Hereford [1995], Salisbury [1996], Bury St Edmunds [1998], Southwell [1998], Chester [2000], Saint-Alban et Rochester [2006]), ce qui n'a fait qu'encourager cette tendance. De plus, on a publié quantité d'«histoires des cathédrales " dans lesquelles l'architecture occupait un ou plusieurs chapitres: Hereford (AYLMER, TILLER, 2000), Winchester (CROOK, 1993), Canterbury (COLLINSON, RAMSEY, SPARKS, 1995), Chichester (HOBBS, 1994), Rochester (yATES, WelSBY, 1996) et Lincoln (owen, 1994), ainsi que pour la Christ Church de Dublin (MILNE, 2000). Il faut aussi mentionner les monographies exclusivement consacrées à l'architecture, comme les récentes publications dont les cathédrales et abbayes de Saint-Alban (ROBERTS, 1993), Rochester (MCALEER, 1999), Salisbury (COCKE, KIDSON, 1993) et Rievaulx (FERGUSSON, HARRISON, 1999) ont fait l'objet. Avec ce dernier ouvrage, Peter Fergusson et Stuart Harrison ont donné une évocation particulièrement marquante de l'une des abbayes anglaises les plus représentatives, qu'ils restituent dans toute l'ampleur de son contexte historique et monastique. Les monographies constituent bien le socle de notre discipline, et elles fournissent une base à partir de laquelle des questionnements plus larges peuvent et doivent être formulés, mais rien de plus.

réticences des universitaires à sortir des limites de leur spécialité sont bien connues, qu'il s'agisse de l'architecture, du vitrail, de la sculpture, de la peinture murale, de la musique ou de tout autre domaine ${ }^{24}$. Les occasions d'échanges interdisciplinaires ne manquent pas, mais peu de chercheurs ont été préparés à une approche globale. P. Binski est l'exception la plus remarquable : son livre sur l'abbaye de Westminster s'aventure bien au-delà de l'histoire de l'architecture, sur le terrain d'une enquête culturelle et idéologique qui s'attache à toutes les formes d'art, ainsi qu'aux idées politiques et religieuses (BINSKI, 1995). Rares sont les édifices qui se prêtent à ce type d'étude. Cependant, même appliquée à des réalisations architecturales plus modestes, l'approche globale peut se révéler féconde. En témoignent deux articles exemplaires sur les chapelles de chantrerie de North Leigh (Oxfordshire; HEARD, 2001) et de Tong (Shropshire ; scotT, 2005), ainsi que l'ouvrage de John Goodall sur l'église et l'hospice d'Ewelme (Oxfordshire; GOODALL, 2001). À Tong, des voûtes en éventail vinrent embellir l'extravagante chapelle funéraire construite entre 1515 et 1519 pour Sir Henry Vernon, et l'ensemble du projet s'inspire vraisemblablement de la chapelle du roi Henry VII à Westminster, comme le montre l'étude de H. Scott, qui s'attache non seulement à l'architecture, mais à chacun des aspects de cette commande, qu'il s'agisse de la sculpture tombale ou des peintures et des figures héraldiques qui l'environnent. L'article de Kate Heard sur la chapelle de Wilcote (1439-1345) qui envisage cette réalisation dans sa totalité, comme une entité artistique et spirituelle, l'avait précédé en ce sens. Ces études appartiennent au groupe relativement restreint des travaux consacrés au Moyen Âge tardif, une période qui a suscité moins d'intérêt (on ne sait pourquoi) que le premier âge gothique ${ }^{25}$.

Depuis longtemps, les historiens s'efforcent de clarifier la relation entre architecture et liturgie ; ce n'est qu'un aspect d'un questionnement plus large concernant la fonction assignée aux édifices, leur fonctionnement effectif et la manière dont ils étaient compris (on trouve une introduction très utile à ce sujet dans DRAPER, 2006). Bien sûr, on s'est beaucoup intéressé à la localisation des autels, des sanctuaires et des clôtures, ainsi qu'à leur environnement architectural. Longtemps, l'extension de beaucoup de cathédrales anglaises dans leur partie orientale fut expliquée par la nécessité d'accroître l'espace pour la circulation autour des autels et des reliques, mais Benjamin 
Nilson, dans une judicieuse mise au point, a critiqué quelques-unes des hypothèses les plus généralement admises à ce sujet (NILSON, 1998). De fait, on peut s'interroger quant au degré d'influence des motifs liturgiques et dévotionnels sur la configuration des principaux lieux de culte, notamment si l'on songe que des structures très dissemblables peuvent se prêter à des liturgies à peu près identiques. Rien n'indique, par exemple, que l'extension de L'usage de Sarum $^{26}$ ait conduit à uniformiser l'architecture des églises ${ }^{27}$. On trouve cependant un cas d'influence liturgique dans l'introduction de galeries intérieures au-dessus du portail Ouest, position en retrait qui permettait aux chœurs de chanter l'office durant les processions du dimanche des Rameaux. Cette disposition, étudiée à Wells et à Salisbury (BLUM, 1986 ; SAMPSON, 1998), a été comparée à des agencements analogues en Irlande et en Écosse. Mais on trouve peu d'exemples d'une telle préméditation : le plus souvent, les pratiques de dévotion se sont adaptées à l'architecture et non l'inverse.

\section{Perspectives de recherches}

\section{Géographies}

Un thème a été amplement ignoré par les chercheurs, celui du développement « colonial» du gothique anglais, en d'autres termes le fait qu'aux environs de 1250 ses styles de construction furent adoptés (ou imposés) dans les îles Britanniques, des Orcades jusqu'au littoral occidental de l'Irlande. Le constat de ce rayonnement vient ajouter une dimension supplémentaire à la question de l'identité « nationale ». La nef de la cathédrale Christ Church de Dublin en est un bon exemple : sa forme très élaborée s'inspire de l'architecture anglaise de l'Ouest (STALLEY, 2000). À Édimbourg, l'abbaye de Holyrood dénote l'influence des édifices du nord de l'Angleterre et notamment de la cathédrale de Lincoln (WILSON, 1984). Au XIII ${ }^{\mathrm{e}}$ siècle, les modèles anglais étaient plus largement répandus qu'ils ne le furent jamais au Moyen Âge. On assiste à un changement spectaculaire au cours des siècles suivants : en divers points de la GrandeBretagne, les constructeurs adoptent alors leurs propres programmes. Ce phénomène a été négligé et personne n'a encore expliqué de manière satisfaisante le développement à partir $\mathrm{du} \mathrm{xV}^{\mathrm{e}}$ siècle, en Écosse et dans une moindre mesure en Irlande, d'une architecture plus proche de celle du continent que de l'Angleterre - à l'exception notable de Richard Fawcett (FAWCETT, 1996 ; FAWCETT, 2002). Ces deux pays ont rejeté le style perpendiculaire : reste à savoir s'ils le firent sciemment ou non (FAWCETT, 2002 ; STALLEY, 1985 ; STALLEY, 1995). Les nombreux éléments décoratifs d'inspiration française (le tracé des fenêtres, le profil des moulures...) dans les travaux de John Morow vers 1400 à l'abbaye de Melrose sont un cas bien connu, mais il est loin d'être unique. Certains auteurs ont tendance à oublier que les mots «anglais » et «britannique » ne sont pas synonymes et que l'architecture britannique ne se réduit pas à l'addition de l'architecture anglaise et de quelques rejetons périphériques du Nord et de l'Ouest ${ }^{28}$. Focalisé sur la notion d'«englishness", Pevsner ne prenait pas en compte certaines parties des îles Britanniques, et il n'était pas le seul ${ }^{29}$. Les expositions londoniennes de 1987 (Age of Chivalry, 1987) et 2003 (Gothic Art for England, 2003) ont renforcé cette tendance anglo-centriste, en laissant l'impression que rien d'important n'avait eu lieu hors des strictes limites de l'Angleterre ${ }^{30}$. Plus largement, on peut s'interroger sur l'insularité de nos études architecturales. Le domaine européen est prospecté quand la 
recherche des origines y conduit, mais les études comparatistes sont encore au berceau ${ }^{31}$.

\section{Le rôle des ordres religieux}

17 Les travaux consacrés récemment à l'architecture cistercienne font ici exception, par l'ouverture géographique et historique de leurs angles d'approche. En 1983, la "Cambridge conference» a inauguré une nouvelle étape des études cisterciennes (NORTON, PARK, 1986) : toute une série d'ouvrages sur les établissements de Cîteaux en Angleterre, en Irlande et plus récemment au Pays de Galles a été publiée depuis lors (FERGUSSON, 1984 ; STALLEY, 1987 ; ROBINSON, 1998 ; KINDER, 2004 ; ROBINSON, 2006). Voilà des années que les chercheurs s'efforcent de mieux saisir la contribution des cisterciens à l'architecture britannique et la part qu'ils ont prise dans ses changements, en particulier dans le contexte du premier âge gothique. Les activités de l'ordre au cours des périodes suivantes ont moins retenu l'attention. Un des mérites de l'ouvrage que David Robinson a consacré aux abbayes galloises tient à sa manière d'envisager les bâtiments cisterciens tout au long du Moyen Âge, en accordant une égale importance à tous les types de construction monastique (ROBINSON, 2006). L'accent n'est pas mis sur le style mais sur l'histoire de l'éclosion des édifices, l'auteur mobilisant à cette fin le moindre document disponible, tous les témoignages archéologiques et architecturaux. Dans bien des cas, les cisterciens influencèrent davantage l'architecture locale du Pays de Galles et de l'Irlande qu'ils ne le firent en Angleterre, et nous attendons depuis longtemps une enquête globale qui évaluerait la contribution de l'ordre pour l'ensemble de la Grande-Bretagne. Mais ils ne méritent pas seulement d'être étudiés pour le rôle actif qu'ils ont joué dans l'histoire du gothique, il convient aussi d'analyser leur influence sur les autres ordres, en particulier les augustins. La façon dont les établissements de Cîteaux se sont étendus, adaptés, restreints dans certains cas, tout cela mérite qu'on s'y arrête. Les travaux dont ils ont déjà fait l'objet démontrent quel parti on peut tirer de l'étude d'un ordre monastique particulier, ou même d'un certain type d'édifice.

18 Il est donc étonnant que les autres ordres soient négligés à ce point. Des recherches sur les édifices franciscains et dominicains, par exemple, aideraient à mieux comprendre l'architecture médiévale britannique dans ses expressions urbaines. Les villes anglaises n'ont pas conservé grand-chose de ces bâtiments, mais les témoignages abondent en Irlande où de nombreux couvents, avec leurs cloitres charmants, ont été conservés à peu près intacts depuis le $\mathrm{Xv}^{\mathrm{e}}$ siècle. Il serait temps de les intégrer à l'enquête.

\section{Tours, flèches, nervures}

Au Moyen Âge, les entreprises des bâtisseurs n'allaient pas sans risques et rares sont les monuments qui n'eurent pas à subir leur part de désastres. Le problème des tours de croisée était particulièrement sensible : le grand effondrement de 1322 qui motiva la construction du célèbre octogone d'Ely ne fut qu'une catastrophe parmi tant d'autres. Les conséquences de ce genre d'accident ont été étudiées à Kirkwall (THURLBY, 1997) ainsi qu'à Lincoln (STALLEY, 2006), mais un travail de synthèse sur les tours de croisée reste encore à écrire. La tour principale et la flèche de Salisbury ont été étudiées par Tatton-Brown (TATTON-BROWN, 1991), mais il est regrettable que l'architecture des tours 
et des flèches n'intéresse d'ordinaire que les seuls érudits locaux, exception faite du travail de Brian O'Callaghan sur les flèches de l'Angleterre médiévale (o'CALLAGHAN, 1997).

Autre négligence, plus surprenante: le domaine des voûtes à nervures demeure en friche. Aucune étude approfondie n'a été publiée depuis la parution de l'ouvrage de Walter Leedy sur les voûtes en éventail (LEEDY, 1980), et les connaissances sur les étonnantes voûtes à liernes des $\mathrm{XIV}^{\mathrm{e}}$ et $\mathrm{XV}^{\mathrm{e}}$ siècles attendent encore d'être mises à jour. Ces nervures, telles que celles qui apparaissent à Durham, sont là pour leur effet visuel plutôt que pour des raisons structurelles, comme le fait comprendre la popularité des voûtes de bois nervurées analysées par Fil Hearn et M. Thurlby : (THURLBY, HEARN, 1997). Si les voûtes de pierre ont été négligées, l'étude des plafonds de bois a bénéficié des progrès de la dendrochronologie, avec des résultats remarquables, notamment à Lincoln et à Salisbury (SIMPSON, FOOT, LITTON, 1986 ; SIMPSON, 1996 ; SIMPSON, LITTON, 1996). Mais les historiens d'art montrent peu d'intérêt pour les travaux concernant les plafonds de bois, qui connaissent un important renouvellement.

\section{Architectes et maîtres maçons}

21 On entend dire parfois que l'histoire de l'architecture médiévale est trop anonyme pour pouvoir véritablement "prendre vie». Voilà plus de trente ans, John Harvey s'est efforcé de répondre à ce reproche d'une histoire "sans homme » en accumulant de nombreuses données d'archive. Les biographies se sont multipliées depuis, pour une bonne part sur la base d'analyses stylistiques (HARVEY, 1972 ; HARVEY, (1954) 1984). Bien que l'approche biographique n'ait jamais fait le fondement de l'histoire du gothique anglais, nous en savons davantage qu'on ne le croit d'ordinaire sur quelques figures majeures, comme William Ramsey, John Wastell ou Henry Janyns le jeune (HARVEY, 1984 ; WILSON, 2003). Des $\mathrm{XII}^{\mathrm{e}}$ et $\mathrm{XIII}^{\mathrm{e}}$ siècles, peu de noms sont parvenus, mais l'étude stylistique nous permet d'identifier bien des individus.

Parmi les autres pistes de recherche, citons l'éducation et la formation des maîtres maçons, leur mode de recrutement, les relations qu'ils entretenaient avec leurs patrons ecclésiastiques et la manière dont les plans étaient conçus. Ce dernier aspect a été mis en lumière dans le cas de Salisbury: Hastings et Draper ont tenté de mieux définir le rôle de l'un des chanoines, Elias de Dereham, qui aurait contribué au plan de la cathédrale du XIII ${ }^{e}$ (HASTINGS, 1997; DRAPER, 2006, p. 45-46). Elias, selon certains, serait moins un maçon de métier qu'une sorte de Villard de Honnecourt. Notre connaissance des maçons médiévaux est tributaire des documents du Moyen Âge tardif et il serait imprudent d'appliquer leurs témoignages à des périodes plus anciennes. En outre, les procédés peuvent varier selon le site. Il suffit de comparer la facture sommaire des maçonneries à la cathédrale de Lincoln et leur impeccable exécution à la cathédrale de Wells pour comprendre que nous avons affaire à deux conceptions très différentes du travail architectural.

Les meilleurs indices d'identification nous sont encore fournis par l'examen des moulurations: leur analyse scientifique est devenue le préalable de tout projet de recherche, depuis le travail précurseur de Richard Morris, qui enseignait naguère à l'université de Warwick et auquel nous sommes tous redevables (MORRIS, 1978-1979; MORRIS, 1990 ; MORRIS, 1992 ; MORRIS, 1996) ${ }^{32}$. On trouvera un parfait exemple de ce type d'approche savante dans une étude sur Thomas de Witney (actif dans les années 
1292-1342), maître maçon qui travailla à la nef de la cathédrale d'Exeter (MORRIS, 1991). L'examen systématique des moulurations a permis de confirmer la remarquable maitrise d'un artisan précis et de révéler l'étendue de son activité dans tout le sud de l'Angleterre. En outre, le nombre des données recueillies par R. Morris et ses élèves a facilité la révision de chronologies communément admises et l'identification de certains modèles régionaux. Suivant son exemple et à partir d'analyses du même type, Linda Monckton a pu clarifier l'histoire de l'abbaye de Sherborne (vers 1380-1450), l'un des plus remarquables monuments de la dernière période gothique, renommé pour le décor spectaculaire de ses voûtes en éventail (MONCKTON, 2000).

Les archéologues de formation ont contribué plus que d'autres à l'histoire de la construction des édifices. Jennifer Alexander, par exemple, a montré quel parti on pouvait tirer d'une étude précise des marques de maçons, des changements d'outillage et des différents types de maçonnerie : c'est ainsi qu'elle a pu établir avec précision les étapes de construction du chœur de Southwell Minster (ALEXANDER, 1996; ALEXANDER, 1998). Des articles d'un grand intérêt ont été consacrés à l'extraction, à la charpenterie, à la ferronnerie et autres métiers dans un certain nombre de publications collectives (PARSONS, 1990 ; BLAIR, RAMSEY, 1991 ; TATTON-BROWN, MUNBY, 1996 ; sur quelques aspects pratiques du travail de construction, il existe une introduction utile dans RODWELL, 1982). Aucune étude sur le rôle de la géométrie, cependant : une lacune surprenante étant donné l'intérêt que cette question a suscité dans le domaine de l'architecture romane et préromane. Parmi les rares exceptions, il faut citer le travail de Peter Kidson sur la cathédrale du XIII siècle à Salisbury (COCKE, KIDSON, 1993) ${ }^{33}$. Les églises gothiques anglaises, construites par ajouts successifs, ne se prêtent pas toujours à ce type d'analyse, mais l'examen de certains éléments particuliers (les piliers ou le tracé des fenêtres, par exemple) peut donner d'intéressants résultats ${ }^{34}$.

L'étude de l'architecture médiévale est à présent une activité multidisciplinaire, et les chercheurs qui s'y consacrent suivent des programmes très variés: archéologie, conservation, histoire de l'art, histoire sociale et religieuse. Dans toutes les disciplines, cependant, on s'accorde sur la nécessité d'aller au-delà de l'architecture matérielle, pour tenter de comprendre l'usage qui était fait des édifices et la façon dont ils étaient perçus et compris. Il a surtout été question ici du premier âge gothique, mais la remarque vaut également pour l'étude de la période suivante. Le livre le plus remarquable pour ce qui concerne l'église paroissiale de la fin du Moyen Âge est sans doute The Stripping of the Altars d'Eamon Duffy (DufFY, [1959] 1992), et il ne s'agit pas d'une étude architecturale mais d'une brillante enquête sur la foi chrétienne telle qu'on la pratiquait en Angleterre à la veille de la Réforme. Ainsi, les historiens de l'architecture se trouvent-ils aujourd'hui confrontés à une stimulante variété d'approches, ce qui les oblige plus que jamais à se poser ces deux questions essentielles : pourquoi étudions-nous l'histoire de l'architecture et pour qui l'écrivonsnous? 


\section{BIBLIOGRAPHIE}

- Age of Chivalry, 1987 : The Age of Chivalry: Art in Plantagenet England, 1200-1400, Jonathan J.G. Alexander, Paul Binski éd., (cat. expo., Londres, Royal Academy of Arts, 1987), Londres, 1987.

- ALEXANDER, 1996 : Jennifer Alexander, « Masons' Marks and Stone Bonding ", dans Tim TattonBrown, Julian Munby éd., The Archaeology of Cathedrals, Oxford, 1996, p. 219-236.

- ALEXANDER, 1998 : Jennifer Alexander, « Southwell Minster Choir: the Evidence of the Masons' Marks ", dans Jennifer Alexander éd., Southwell and Nottinghamshire, Medieval Art, Architecture, and Industry, (British Archaeological Association Conference Transaction, XXI), Londres, 1998, p. 44-59.

- ALEXANDER, 1998 : Jonathan J. G. Alexander, « Medieval art and the modern nationalism », dans Gale R. Owen-Crocker, Timothy Graham éd., Medieval Art: Recent Perspectives. A memorial tribute to C. R. Dodwell, Manchester, 1998, p. 206-223.

- AYLMER, TILLER, 2000 : Gerald Aylmer, John Tiller éd., Hereford Cathedral. A History, Londres, 2000.

- BINSKI, 1995 : Paul Binski, Westminster Abbey and the Plantagenets: Kingship and the Representation of Power 1200-1400, Londres/New Haven, 1995.

- BINSKI, 2005 : Paul Binski, Becket's Crown. Art and Imagination in Gothic England 1170-1300, Londres/ New Haven, 2005.

- BLAIR, RAMSEY, 1990 : John Blair, Nigel L. Ramsey éd., English Medieval Industries, Craftsmen, Techniques, Products, Londres, 1991.

- BLUM, 1986 : Pamela Z. Blum, «Liturgical Influences on the Design of the West Front at Wells and Salisbury », dans Gesta, XXV/1, 1986, p. 145-150.

- BOND, 1906 : Francis Bond, Gothic Architecture in England, Londres, 1906.

- BONY, 1949 : Jean Bony, «French Influences on the Origins of English Gothic Architecture », dans Journal of the Warburg and Courtauld Institutes, XII, 1949, p. 1-15.

- BRAKSPEAR, 1931 : Harold Brakspear, «A West Country School of Masons », dans Archaeologia, 81, 1931, p. $1-18$

- BREDEKAMP, 2003 : Horst Bredekamp, « A neglected tradition? Art history as Bildwissenschaft », dans Michael F. Zimmermann éd, The Art Historian. National Traditions and Institutional Practices, New Haven, 2003, p. 147-159.

- COCKE, KIDSON, 1993 : Thomas Cocke, Peter Kidson, Salisbury Cathedral Perspectives on the Architectural History, Londres, 1993.

- COLDSTREAM, 1994 : Nicola Coldstream, The Decorated Style, Architecture and Ornament 1240-1360, Londres, 1994.

- COLDSTREAM, 2004 : Nicola Coldstream, « Nikolaus Pevsner and medieval studies in Britain », dans DRAPER, 2004, p. 357-360.

- COLLINSON, RAMSEY, SPARKS, 1995 : Patrick Collinson, Nigel Ramsey, Margaret Sparks éd., A History of Canterbury Cathedral, Oxford, 1995. 
- Colvin, 1963 : Howard Colvin éd., A History of the King's Works: The Middle Ages, 3 vol., Londres, 1963.

- CRоOK, 1993 : John Crook éd., Winchester Cathedral. Nine Hundred Years 1093-1993, Chichester, 1993.

- CROSSLEY, 2001 : Paul Crossley, compte rendu de NUSSBAUM, 2000, dans Journal of the Society of Architectural Historians, 60/1, 2001, p. 95.

- DAVIDSON CRAGOE, 2001 : Carol Davidson Cragoe, « Reading and Rereading Gervase of Canterbury », dans Journal of the British Archaeological Association, 154, 2001, p. 40-53.

- DAVIS, 2001 : Michael T. Davis, compte rendu de FERGUSSON, HARRISON, 1999, dans Journal of the Society of Architectural Historians, 60/1, 2001, p. 87-93.

- DRAPER, 1983 : Peter Draper, « William of Sens and the original design of the choir termination of Canterbury Cathedral 1175-1179 ", dans Journal of the Society of Architectural Historians, 53/3, 1983, p. 238-248.

- DRAPER, 1999 : Peter Draper, « St David's Cathedral: Provincial or Metropolitan? », dans Fabienne Joubert, Dany Sandron, Pierre, lumière, couleur : études d'histoire de l'art du Moyen Âge en honneur d'Anne Prache, Paris, 1999, p. 103-116.

- DRAPER, 2004 : Peter Draper éd., Reassessing Nikolaus Pevsner, Aldershot, 2004.

- DRAPER, 2006 : Peter Draper, The Formation of English Gothic. Architecture and Identity, Londres/New Haven, 2006.

- DUfFy, (1959) 1992 : Eamon Duffy, The Stripping of the Altars: Traditional Religion in England c.1400-c. 1580, (Milan, 1959), Londres/New Haven, 1992.

- ECo, 1986 : Umberto Eco, Art and Beauty in the Middle Ages, Londres/New Haven, 1986 (Milan, 1987 ; trad. fr. : Art et beauté dans l'esthétique médiévale, Paris, 1997).

- EMERY, 1999 : Anthony Emery, Greater Medieval Houses of England and Wales 1300-1500, III, Southern England, Cambridge, 2006.

- FAWCETT, 1996 : Richard Fawcett, «Architectural links between Scotland and the Low Countries in the later middle ages ", dans Elizabeth de Bièvre éd., Utrecht, Britain and the Continent, Archaeology, Art and Architecture, (British Archaeological Association Conference Transactions, XVIII), Londres, 1996, p. 172-182.

- FAWCETT, 2002 : Richard Fawcett, Scottish Medieval Churches, Architecture and Furnishings, Stroud, 2002.

- FERGUSSON, 1971 : Peter Fergusson, « Roche Abbey: the source and date of the eastern remains », dans Journal of the British Archaeological Association, 34, 1971, p. 30-42.

- FERGUSSON, 1975 : Peter Fergusson, « The South Transept Elevation of Byland Abbey », dans Journal of the British Archaeological Association, 38, 1975, p. 155-176.

- FERGUSSON, 1984 : Peter Fergusson, Architecture of Solitude, Cistercian Abbeys in Twelfth-Century England, Princeton, 1984. 
- FERGUSSON, HARRISON, 1999 : Peter Fergusson, Stuart Harrison, Rievaulx Abbey. Community Architecture, Memory, Londres/New Haven, 1999.

- FERNIE, 2003 : Eric Fernie, « The History of Art and Archaeology in England Now », dans Michael F. Zimmermann éd., The Art Historian. National Traditions and Institutional Practices, New Haven, 2003, p. 160-166.

- FERNIE, CROSSLeY, 1990 : Eric Fernie, Paul Crossley éd., Medieval Architecture and its Intellectual Context, Londres, 1990.

- GOMME, 2006 : Andor Gomme, compte rendu de EMERY, 1999, dans Society of Architectural Historians of Great Britain Newsletter, 89, fall, 2006, p. 18.

- GOODALL, 2001 :John Goodall, God's House at Ewelme: Life, Devotion and Architecture in a FifteenthCentury Almshouse, Aldershot, 2001.

- Gothic Art for England..., 2003 : Gothic Art for England, 1400-1547, Richard Marks, Paul Williamson éd., (cat. expo., Londres, Victoria and Albert Museum, 2003), Londres, 2003.

- GRANT, 1991 : Lindy Grant, « Gothic Architecture in Southern England and the French Connection in the Early Thirteenth century ", dans Simon Lloyd, Peter Coss éd., Thirteenth Century England, III, Woodbridge, 1991, p. 113-126.

- GRANT, 1994 : Lindy Grant, « Architectural relationships between England and Normandy, 1100-1204 », dans David Bates, Anne Curry éd., England and Normandy in the Middle Ages, Londres, 1994, p. 117-129.

- HARRISON, 1990 : Stuart Harrison, Byland Abbey, Londres, 1990.

- HARRISON, 2003 : Stuart Harrison, « Elgin Cathedral: The Western Rose Reconstructed ", dans Journal of the British Archaeological Association, 156, 2003, p. 138-149.

- HARRISON, BARKER, 1987 : Stuart Harrison, Paul Barker, « Byland Abbey, North Yorkshire: The west front and rose window reconstructed », dans Journal of the British Archaeological Association, 140, 1987, p. 134-151.

- HARRISON, BARKER, 1999 : Stuart Harrison, Paul Barker, « Ripon Minster: An Archaeological Analysis and Reconstruction of the $12^{\text {th }}$ Century Church ", dans Journal of the British Archaeological Association, 152, 1999, p. 49-78.

- HARVEY, 1972 : John Hooper Harvey, The Medieval Architect, Londres, 1972.

- HARVEY, 1978 : John Hooper Harvey, The Perpendicular Style 1330-1485, Londres, 1978.

- HARVEY, (1954) 1984 : John Hooper Harvey, English Medieval Architects: A Biographical Dictionary down to 1550, (Londres, 1954), Gloucester, 1984.

- HASTINGS, 1997 : Adrian Hastings, Elias of Dereham. Architect of Salisbury Cathedral, Salisbury, 1997.

- HAWARD, 1993 : Birkin Haward, Suffolk Medieval Church Arcades, 1150-1550, Ipswich, 1993.

- HEARD, 2001 : Kate Heard, « Death and Representation in the Fifteenth Century: The Wilcote Chantry Chapel at North Leigh », dans Journal of the British Archaeological Association, 154, 2001, p. 134-149. 
- HEARN, 1983 : Millard Fillmore Hearn, Ripon Minster: The Beginning of the Gothic Style in Northern England, (Transactions of the American Philosophical Society, 73, part. 6), Philadelphia, 1983.

- HEARN, 1994 : Millard Fillmore Hearn, « Canterbury Cathedral and the Cult of Becket », dans The Art Bulletin, 75, 1994, p. 19-52.

- HENIG, MCNEILL, 2006 : Martin Henig, John McNeill éd., The Medieval Cloister in England and Wales, numéro spécial du Journal of the British Archaeological Association, 159, 2006.

- HESLOP, 2000 : Thomas Alexander Heslop, « Art and Nature at St Hugh's Choir at Lincoln », dans MITCHELL, 2000, p. 60-74.

- HESLOP, 2005 : Thomas Alexander Heslop, « Swaffham Parish Church: Community Building in Fifteenth-Century Norfolk », dans Christopher Harper-Bill éd., Medieval East Anglia, Woodbridge, 2005, p. 246-271.

- HoвBs, 1994 : Mary Hobbs éd., Chichester Cathedral, An Historical Survey, Chichester, 1994.

- HOEY, 1986 : Lawrence Hoey, «Pier Alternation in Early English Gothic Architecture », dans Journal of the British Archaeological Association, 139, 1986, p. 45-67.

- HOEY, 1987 : Lawrence Hoey, « Piers Versus Vault Shafts in Early English Gothic Architecture », dans Journal of the Society of Architectural Historians, 46, 1987, p. 241-264.

- HOEY, 1994 : Lawrence Hoey, « Pier Design in Early Gothic Architecture in East-Central Scotland c.1170-1250 ", dans John Higgitt éd., Medieval Art and Architecture in the Diocese of St Andrews, (British Archaeological Association Conference Transactions, XIV), Londres, 1994, p. 84-98.

- HOEY, 1995 : Lawrence Hoey, « New Studies on Canterbury Cathedral », dans Avista Forum, 9 , 1995, p. 6-9.

- HOEY, 2000 : Lawrence Hoey, Tradition, Innovation and Creative Adaptation: The Medieval Rebuilding of English Church Architecture. 1066-1530, [non publié], 2000.

- HOWARD, 2002 : Deborah Howard, « Teaching Architectural History in Great Britain and Australia: Local Conditions and Global Perspectives », dans Journal of the Society of Architectural Historians, 61/3, 2002, p. 346-354.

- KIDSON, 1993 : Peter Kidson, « Gervase, Becket and William of Sens », dans Speculum, 68, 1993, p. $969-991$.

- KIDSON, MURRAY, THOMPSON, 1962 : Peter Kidson, Peter Murray, Paul Thompson, A History of English Architecture, Harmondsworth, 1965.

- KINDER, 2004 : Terryl N. Kinder éd., Perspectives for an Architecture of Solitude, Essays on Cistercians, Art and Architecture in Honour of Peter Fergusson, Turnhout, 2004.

- LEEDY, 1980 : Walter C. Leedy, Fan Vaulting: A Study of Form, Technology, and Meaning, Londres, 1980.

- LEHMANN-BROCKHAUS, 1955 : Otto Lehmann-Brockhaus, Lateinische Schriftquellen zur Kunst in England, Wales und Schottland, vom Jahre 901 bis zum Jahre 1307, Munich, 1955. 
- MAIR, 1982 : Rosalind Mair, « The Choir Capitals of Canterbury Cathedral, 1174-84 », dans Nicola Coldstream, Peter Draper éd., Medieval Art and Architecture at Canterbury before 1220, (British Archaeological Association Conference Transactions, v), Londres, 1982, p. 56-66.

- MALONE, 1973 : Carolyn Marino Malone, West English Gothic Architecture 1175-1250, (PhD, University of California, Berkeley), 1973.

- MALONE, 1984 : Carolyn Marino Malone, «Abbey Dore: English versus French Design », dans Meredith P. Lillich éd., Studies in Cistercian Art and Architecture, 2, 1984, p. 50-75.

- MALONE, 2004a : Carolyn Marino Malone, « Cistercian Design in the Choir and Transept of Wells Cathedral », dans KINDER, 2004, p. 351-368.

- MALONE, 2004b : Carolyn Marino Malone, Façade as Spectacle: Ritual and Ideology at Wells Cathedral, Leyde/Boston, 2004.

- MCALEER, 1999 : John Philip McAleer, Rochester Cathedral 604-1540, Toronto, 1999.

- MILNE, 2000 : Kenneth Milne éd., Christ Church Cathedral Dublin, A History, Dublin, 2000.

- MITChell, 2000 : John Mitchell éd., England and the Continent in the Middle Ages: Studies in Memory of Andrew Martindale, (Harlaxton Medieval Studies, VII), Stamford, 2000.

- MONCKTON, 1999 : Linda Monckton, Late Gothic Architecture in South West England, (PhD, University of Warwick), 1999.

- MONCKTON, 2000 : Linda Monckton, « The Late Medieval Rebuilding of Sherborne Abbey: A Reassessment », dans Architectural History, 43, p. 88-112.

- MORRIS, 1978-1979 : Richard K. Morris, « The development of later Gothic mouldings in England c.1250-1400 », dans Architectural History, 21, 1978, p. 18-57 et 22, 1979, p. 1-48.

- MORRIS, 1990 : Richard K. Morris, « Mouldings and the Analysis of Medieval Style », dans FERNIE, CROSSLEY, 1990, p. 239-248.

- MORRIS, 1991 : Richard K. Morris, « Thomas of Witney at Exeter, Winchester and Wells », dans Francis Kelly éd., Medieval Art and Architecture at Exeter Cathedral, (British Archaeological Association Conference Transactions, XI), Londres, 1991, p. 57-84.

- MORRIS, 1992 : Richard K. Morris, « An English glossary of medieval mouldings: with an introduction to mouldings c.1040-1240 », dans Architectural History, 35, 1992, p. 1-17.

- MORRIS, 1996 : Richard K. Morris, « Mouldings in Medieval Cathedrals », dans TATTON-BROwN, MUNBY, 1996, p. 211-217.

- MORRIS, 2003 : Richard K. Morris, compte rendu dans Journal of the British Archaeological Association, 156, 2003, p. 188-190.

- NILSON, 1998 : Benjamin John Nilson, Cathedral Shrines of Medieval England, Woodbridge, 1998. - NORTON, PARK, 1986 : Christopher Norton, David Park éd., Cistercian Art and Architecture in the British Isles, Cambridge, 1986.

- NUSSBAUM, 1994 : Norbert Nussbaum, Deutsche Kirchenbaukunst der Gotik, Darmstadt, 1994. - NUSSBAUM, 2000 : Norbert Nussbaum, German Gothic Church Architecture, Londres/New Haven, 2000. 
- o'CALLAGHAN, 1995 : Brian O'Callaghan, « An Analysis of the Architecture of the Cistercian Church at Abbey Dore ", dans David Whitehead éd., Medieval Art, Architecture and Archaeology at Hereford, (British Archaeological Association Conference Transactions, Xv), Londres, 1995, p. 94-104.

- O'CALLAGHAN, 1997 : Brian O'Callaghan, «English Medieval Spires: Design and meaning ", dans Frank Salmon éd., Gothic and the Gothic Revival, Papers from the $26^{\text {th }}$ Annual Symposium of the Society of Architectural Historians of Great Britain, Manchester, 1998, p. 45-56.

- O'KEEFE, 2003 : Tadhg O'Keeffe, Romanesque Ireland: Architecture and Ideology in the Twelfth Century, Dublin, 2003.

- O'KEEFE, 2006 : Tadhg O'Keeffe, « Wheels of words, networks of knowledge: Romanesque scholarship and Cormac's Chapel », dans Damian Bracken, Dagmar O'Riain-Raedel éd., Ireland and Europe in the Twelfth Century, Dublin, 2006, p. 257-269.

- OWEN, 1994 : Dorothy Owen éd., A History of Lincoln Minster, Cambridge, 1994.

- PARSONS, 1990 : David Parsons éd., Stone: Quarrying and Building in England A.D. 43-1525, Chichester, 1990.

- PEVSNER, 1951 : Nikolaus Pevsner, The Buildings of England series, Harmondsworth, 1951-1974.

- PEVSNER, METCALF, 1985 : Nikolaus Pevsner, Priscilla Metcalf, The Cathedrals of England, 2 vol., Harmondsworth, 1985.

- PLATT, 1990 : Colin Platt, The Architecture of Medieval Britain, Londres/New Haven, 1990.

- ROBERTS, 1977 : Eileen Roberts, « Moulding analysis and architectural research: The late Middle Ages », dans Architectural History, 20, 1977, p. 5-13.

- ROBERTS, 1993 : Eileen Roberts, The Hill of the Martyr, An Architectural History of St Albans Abbey, Dunstable, 1993.

- ROBINSON, 1998 : David M. Robinson éd., The Cistercian Abbeys of Britain: Far from the Concourse of Men, Londres, 1998.

- ROBINSON, 2006 : David M. Robinson, The Cistercians in Wales, Architecture and Archaeology 1130-1545, Londres, 2006.

- RODWELL, 1982 : Warwick Rodwell, The Archaeology of the English Church, Londres, 1982.

- SAMPSON, 1998 : Jerry Sampson, Wells Cathedral West Front, Construction, Sculpture and Conservation, Stroud, 1998

- SAUERLÄNDER, 1995 : Willibald Sauerländer, «Integration: A Closed or Open Proposal? », dans Virginia Chieffo Raguin, Kathryn Brush, Peter Draper éd., Artistic Integration in Gothic Buildings, Toronto, 1995, p. 3-18.

- ScOTT, 2005 : Heather Gilderdale Scott, « 'this little westminster': The Chantry-Chapel of Sir Henry Vernon at Tong, Shropshire ", dans Journal of the British Archaeological Association, 158, 2005, p. 46-81.

- SIMPSON, 1996 : W. Gavin Simpson, « Documentary and Dendrochronological Evidence for the Building of Salisbury Cathedral », dans Laurence Keene, Thomas Cocke éd., Medieval Art and 
Architecture at Salisbury Cathedral, (British Archaeological Association Conference Transactions, XVII), 1996, p. 10-20.

- SIMPSON, FOOT, LITTON, 1986 : W. Gavin Simpson, N. Foot, Clifford D. Litton, « The High Roofs of the East End of Lincoln Cathedral », dans Thomas Alexander Heslop, Veronica Sekules éd., Medieval Art and Architecture at Lincoln Cathedral, (British Archaeological Association Conference Transactions, VIII), Londres, 1986, p. 47-74.

- SIMPSON, LITTON, 1996 : W. Gavin Simpson, Clifford D. Litton, « Dendrochronology in Cathedrals », dans TATTON-BROWN, MUNBY, 1996, p. 183-209.

- STALLEY, 1985 : Roger Stalley, « Irish Gothic and English Fashion », dans James Lydon éd., The English in Medieval Ireland, Dublin, 1984, p. 65-86.

- STALLEY, 1987 : Roger Stalley, The Cistercian Monasteries of Ireland, Londres/New Haven, 1987.

- STALLEY, 1990 : Roger Stalley, « Gaelic friars and Gothic design », dans FERNIE, CROSSLEY, 1990, p. 191-202.

- STALLEY, 1994 : Roger Stalley, «Ireland and Scotland in the Later Middle Ages », dans John Higgitt éd., Medieval Art and Architecture in the Diocese of St Andrews, (British Archaeological Association Conference Transactions, XIV), Londres, 1994, p. 108-117.

- STALLEY, 1995 : Roger Stalley, « Choice and Consistency: the early Gothic architecture of Selby Abbey ", dans Architectural History, 38, 1995, p. 1-24.

- STALLEY, 2000 : Roger Stalley, "The construction of the medieval cathedral, c.1030-1250 ", dans MILNE, 2000, p. 53-74.

- STALLEY, 2002 : Roger Stalley, « The Architecture of St David's Cathedral: Chronology, catastrophe and design », dans Antiquaries Journal, 82, 2002, p. 13-46.

- STALLEY, 2006 : Roger Stalley, «'Lapides Reclamabunt': Art and engineering at Lincoln in the thirteenth century », dans Antiquaries Journal, 86, 2006, p. 131-147.

- STEIBER, 2003 : Nancy Steiber, « Editorial: Architecture between Disciplines », dans Journal of the Society of Architectural Historians, 61/2, 2003, p. 176.

- TATTON-BROWN, 1991 : Tim Tatton-Brown, « Building the Tower and Spire of Salisbury Cathedral », dans Antiquity, 65, 1991, p. 74-96.

- TATTON-BRown, MUNBY, 1996 : Tim Tatton-Brown, Julian Munby, (The Archaeology of Cathedrals, Oxford University Committee for Archaeology, Monograph 42), Oxford, 1996.

- THURLBY, 1994 : Malcolm Thurlby, «St Andrews Cathedral-Priory and the beginnings of Gothic architecture in Northern Britain », dans John Higgitt éd., Medieval Art and Architecture in the Diocese of St Andrews, (British Archaeological Association Conference Transactions, XIV), Londres, 1994, p. 47-60.

- THURLBY, 1997 : Malcolm Thurlby, « Aspects of the architectural history of Kirkwall Cathedral », dans Proceedings of the Society of Antiquaries of Scotland, 127, 1997, p. 855-888.

- THURLBY, 2000 : Malcolm Thurlby, « Roger Pont l'Eveque, Archbishop of York (1154-81) and French Sources for the Beginning of Gothic in Northern Britain », dans MITCHELL, 2000, p. 35-47. 
- THURLBY, HARRISON, 1997 : Malcolm Thurlby, Stuart Harrison, « An Architectural History », dans Ron Shoesmith, Ruth Richardson éd., A Definitive History of Dore Abbey, Little Logaston, 1997, p. 45-62.

- THURLBY, HEARN, 1997 : Malcolm Thurlby, Millard Fillmore Hearn, « Previously Undetected

Wooden Ribbed Vaults in Medieval Britain ", dans Journal of the British Archaeological Association, 150, 1997, p. 48-58.

- THURLBY, KUSABA, 1991 : Malcolm Thurlby, Yoshio Kusaba, « The Nave of St Andrew at Steyning: A Case Study Variety in Design in Twelfth-Century Architecture in Britain », dans Gesta XXX/2, 1991, p. 163-175.

- WATKIN, 1977 : David Watkin, Morality and Architecture: The Development of a Theme in Architectural History and Theory from the Gothic Revival to the Modern Movement, Oxford, 1977.

- WEBB, 1965 : Geoffrey Webb, Architecture in Britain: The Middle Ages, Harmondsworth, 1965.

- WILSON, 1975 : Christopher Wilson, « The Sources of the Late Twelfth-Century Work at Worcester Cathedral », dans Alan Borg éd., Medieval Art and Architecture at Worcester Cathedral, (British archaeological Association Conference Transactions, I) 1975, Worcester, p. 81-90.

- WILSON, 1984 : Christopher Wilson, « Holyrood: Abbey Church », dans John Gifford, Colin McWilliam, David Walker éd., The Buildings of Scotland: Edinburgh, Harmondsworth, 1984, p. 130-141.

- WILSON, 1986 : Christopher Wilson, « The Cistercians as 'missionaries of Gothic' », dans NORTON, PARK, 1986, p. 86-116.

- WILSON, 2003 : Christopher Wilson, « Excellent, New and Uniform, Perpendicular Architecture c. 1400-1547 », dans Richard Marks, Paul Williamson éd., Gothic Art for England, 1400-1547, Londres, 2003, p. 98-119.

- WILSON, 2004 : Christopher Wilson, « Lausanne and Canterbury: A 'Special Relationship' Reconsidered ", dans Peter Kurmann, Martin Rohde éd., Die Kathedrale von Lausanne und ihr Marienportal im Kontext der europäischen Gotik, Berlin/New York, 2004, p. 89-124.

- YATES, WELSBY, 1996 : Nigel Yates, Paul A. Welsby, Faith and Fabric, A History of Rochester Cathedral 604-1994, Woodbridge, 1996.

\section{NOTES}

1. Parmi les diverses réévaluations dont l'œuvre de N. Pevsner a récemment fait l'objet, voir, pour le domaine médiéval, COLDSTREAM, 1994.

2. Une anthologie en deux volumes reprend les articles de N. Pevsner sur les cathédrales, précédemment publiés dans Buildings of England (PEVSNER, METCALF, 1985). L'ensemble constitue l'une des introductions les plus accessibles dont nous disposions pour le gothique anglais.

3. Mentionnons aussi les chapitres de Peter Kidson dans la première section de KIDSON, MURRAY, THOMPSON, 1965

4. « ...every few generations it is necessary, in the light of such accumulated scholarship, not only to revise our views of such great subjects as these (medieval buildings), but to rewrite the history of an art in the light of the preoccupations of a new generation ". 
5. Nous aurions grand besoin d'une étude nouvelle pour la période du Moyen Âge tardif comme pour le style "perpendiculaire ", sur lequel le livre de John Harvey est difficile à suivre : voir HARVEY, 1978.

6. Sur les rapports de l'histoire de l'architecture avec les autres disciplines, et notamment l'archéologie, voir l'étude pénétrante de HOWARD, 2002.

7. Mais le propre travail de T. O'Keefe sur l'architecture romane est extrêmement conventionnel : voir O'KEEFE, 2003. Il ne s'avise pas que les historiens d'art ont adopté le modèle qu'il préconise par ailleurs : voir BINSKI, 1995 ; BINSKI, 2005.

8. "...obscured the fundamental purpose of medieval house development - social distinction, greater privacy, and more elaborate lifestyles ».

9. Sur les églises paroissiales, quantité de guides ont paru, recyclant pour la plupart des données rebattues; les spécialistes ne se sont guère exercés dans ce domaine. Une exception cependant : HESLOP, 2005.

10. Cette séparation entre églises et châteaux inquiétait déjà Geoffrey Webb voilà cinquante ans : WEBB, 1965, p. xix-Xx.

11. Certains considèrent que le système de reconnaissance académique, qui tend à privilégier les recherches de fin d'études consacrées aux «grands » monuments, est en partie responsable de cet état de fait.

12. «...our topics have diverged from those that at one point seemed to define architectural history: heroic narratives of architects, celebrations of national heritage, justifications for contemporary architectural polemics, or subjects that reinforce the formation of canons", STEIBER, 2003, p. 176.

13. De ces trois termes, « perpendiculaire » est le plus utile pour cerner une démarche stylistique cohérente.

14. En adoptant pour cadre chronologique les années 1200-1400, les organisateurs de l'exposition The Age of Chivalry ont en quelque sorte éludé la terminologie de Rickman, bien que l'un des contributeurs du catalogue vienne redonner ensuite un second souffle à la notion de decorated style : COLDSTREAM, 1994. Il est intéressant de constater que les périodes suivantes répondent à des désignations stylistiquement neutres (Tudor, Jacobean, etc.) et échappent à ces classifications malheureuses par type d'édifices.

15. «...most of our studies of Early and High Gothic architecture have been dominated by the positivistic belief in a linear chronology readable as schema of unambiguous characteristics and identification marks ».

16. Lindy Grant note, par exemple, des points communs avec Arras (colonnes élancées, décor végétal) et l'aire de la Somme et du Boulonnais (nervures de voûtes dentelées, abaques circulaires, chapiteaux sculptés).

17. Jean Bony a soulevé l'hypothèse de sa contribution au plan de la cathédrale de Lausanne : voir BONY, 1957-1958, p. 47. Sur ce sujet, on peut lire à présent WILSON, 2004.

18. «...shows every sign of having been designed by an architect from Picardy or French Flanders ".

19. Au moment de sa mort, en 2000 , Lawrence Hoey venait d'achever le premier jet d'un ouvrage qui devait retracer ce processus de reconstruction ininterrompu à travers l'histoire de l'architecture ecclésiastique.

20. « ...spreads wide its wings like the feathered birds as if in flight, [...] strikes the clouds, though resting on its solid columns".

21. Le goût de la varietas était un héritage de l'époque romane; voir par exemple les piliers de la cathédrale de Rochester, ainsi que les ornements de Steyning et de la cathédrale Saint-David: THURLBY, KUSABA, 1991 ; STALLEY, 2002. 
22. Pour l'emploi de ces termes, voir les nombreux extraits publiés par LEHMAN-BROCKHAUS, 1955. La varietas était très prisée, mais l'expression s'appliquait davantage aux couleurs et aux gemmes qu'à l'architecture. Pour une introduction aux définitions du beau les plus établies, voir ECO, 1986. 23. À la suite d'un différend avec Jean sans Terre sur la nomination de l'archevêque de Canterbury, le pape Innocent III jeta l'interdit sur le royaume d'Angleterre, le privant ainsi de la célébration des sacrements et du culte.

24. Il va sans dire que les spécialistes se retrouvent fréquemment et qu'on ne manque pas d'excellents ouvrages où leurs travaux sont publiés côte à côte - au sens propre : en parallèle, bien plus souvent qu'en collaboration.

25. Comme C. Wilson l'a remarqué, même dans le domaine des plans d'église, la somme des recherches relatives aux églises paroissiales du Moyen Âge tardif est dérisoire: WILSON, 2003, p. 113. Parmi les rares exceptions, HAWARD, 1993, et MONCKTON, 1999.

26. L'Usage de Sarum est une compilation des offices liturgiques réalisées par Osmond de Salisbury (chancelier de Guillaume le Conquérant de 1072 à 1077 et évêque de Salisbury de 1078 jusqu'à sa mort en 1099) pour établir l'uniformité du culte. Amélioré au XIII ${ }^{\mathrm{e}}$ siècle, cet usage s'impose alors rapidement à toute l'Angleterre.

27. Cependant, l'Usage de Sarum a peut-être influencé l'agencement de la cathédrale Saint-Patrick de Dublin.

28. Comme le Dr Bruce Campbell de la Queen's University de Belfast me le faisait remarquer, notre histoire économique dénote la même déformation anglocentriste.

29. La monumentale History of the Kings Works (COLvin, 1963), ignore les châteaux royaux d'Irlande. Nicola Coldstream a noté que l'enthousiasme de Pevsner pour l'englishness ne s'étendait pas aux autres parties du Royaume-Uni (COLDSTREAM, 2004, p. 114). Un constat similaire dans ALEXANDER, 1998, p. 206 : «L'emploi qu'il faisait des termes England/english, comme c'était souvent le cas dans les discours de l'époque, subsumait de fait les autres parties des îles Britanniques, mais le terme british, dans son acception aujourd'hui la plus courante, n'est pas moins dommageable, ayant pour effet de dissimuler ou d'effacer l'existence des peuples du Pays de Galles, de l'Écosse et de l'Irlande " ( His use of England/English, like much of the discourse of that time, subsumes by implication other parts of the British Isles, though the more frequent use of the term British today probably is just as damaging in concealing or erasing the existence of the peoples of Wales, Scotland and Ireland »).

30. La carte publiée dans le catalogue de l'exposition The Age of Chivalry (1987) laisse la totalité de l'Écosse en blanc. L'Irlande n'y apparaît même pas.

31. La décision de la British Archaeological Association d'organiser une année sur deux sa conférence en dehors de la Grande-Bretagne constitue cependant un développement prometteur. Ces dernières années, elles se sont tenues à Rouen, Utrecht, Angers et Prague.

32. Voir aussi les importantes contributions de ROBERTS, 1977. Les moulurations des édifices d'Écosse ont été étudiées par Richard Fawcett : par exemple FAWCETT, 2002.

33. Pour l'étude des proportions d'un couvent irlandais, voir STALLEY, 1990.

34. Un travail de doctorat en cours, consacré au tracé des fenêtres des édifices irlandais, se réfère constamment à la géomatique, technique qui, à l'aide de l'informatique, élabore et analyse des reconstitutions en trois dimensions. 


\section{RÉSUMÉS}

L'étude de l'architecture gothique anglaise est aujourd'hui pluridisciplinaire : des chercheurs de formations variées enquêtent sur ce domaine en se servant de méthodes et de techniques différentes les unes des autres. Cet article prend la mesure des forces et des limites de la recherche récente, en mettant l'accent sur la nécessité d'un nouveau cadre conceptuel dans lequel serait ressaisie l'écriture de l'histoire traditionnelle. Il subsiste une incertitude concernant l'orientation à prendre par l'histoire de l'architecture : doit-elle se concentrer principalement sur le style et les monuments prestigieux ou s'intéresser également à tous les bâtiments, quelle que soit leur nature et leur statut? L'article passe en revue certains de ces points à la lumière des publications récentes : de quelle manière et pour quelles raisons l'Angleterre a-t-elle produit un gothique singulier? Dans quelle mesure l'architecture anglaise reflète-t-elle le sentiment de plus en plus prégnant d'une identité nationale? Et comment les bâtiments étaient-ils perçus et utilisés par les contemporains? Ainsi la dimension coloniale du gothique anglais, telle qu'elle est perceptible par exemple en Écosse et en Irlande, est-elle mise en lumière dans ses grandes lignes, et peut-on conclure sur la nécessité d'une approche plus européenne et moins anglo-centrique.

The study of English Gothic architecture is now a multi-disciplinary occupation, as scholars from various backgrounds apply different methodologies and techniques. This article considers the strengths and limitations of recent scholarship, stressing the need for new conceptual frameworks to replace the traditional linear narrative. There is still uncertainty about whether the history of architecture should be concerned principally with style and the elite monuments or with buildings of every type and status. The article goes on to review some pertinent issues in the light of recent publications: how and why did England formulate an alternative Gothic, to what extent did English architecture reflect a growing sense of national identity, and how were the buildings used and understood by contemporaries? It highlights the colonial dimension of English Gothic as reflected in Scotland and Ireland, and concludes by stressing the need for a more European and less anglo-centric approach.

Die Erforschung der britischen Gotikarchitektur ist heute pluridisziplinär. Wissenschaftler aus verschiedenen Bereichen bearbeiten dieses Thema mit Hilfe von sehr unterschiedlichen Methoden und Techniken. Dieser Artikel beleuchtet die Stärken und Grenzen der jüngeren Forschung, indem er die Notwendigkeit eines neuen konzeptuellen Rahmens zur Erfassung der traditionellen Geschichtsschreibung herausstellt. Unsicherheit bleibt jedoch in Bezug auf die zu wählende Richtung in der Architekturgeschichte. Sollte sie sich vorrangig auf den Stil und die renommierten Bauten konzentrieren oder jegliche Bauwerke, welcher Art oder Bedeutung auch immer, mit einbeziehen? Der Artikel vollzieht einige dieser Punkte anhand der jüngeren Publikationen nach. Auf welche Weise und aus welchen Gründen hat sich in England eine eigene, singuläre Gotik entwickelt? Inwiefern spiegelt die britische Architektur das immer stärker werdende Gefühl einer nationalen Identität wider? Wie wurden diese Bauwerke von ihren Zeitgenossen wahrgenommen und benutzt? In diesem Sinne kommt der koloniale Kontext der britischen Gotik weitestgehend zum Tragen, wie er sich zum Beispiel in Schottland und Irland ausgeprägt hat, und verweist damit auf die Notwendigkeit einer stärker europäisch und weniger englisch ausgerichteten Herangehensweise.

Lo studio dell'architettura gotica inglese è ai giorni nostri un insegnamento pluridisciplinare ; ricercatori dalla diversa formazione indagano su questo campo utilizzando spesso metodi e tecniche disparati. Questo articolo valuta i punti di forza e i limiti della ricerca recente insistendo sulla necessità di una nuova impostazione concettuale che possa sostituire la linea interpretativa 
tradizionale. Persiste un'incertezza che riguarda l'orientamento che deve prendere la storia dell'architettura: se essa si debba concentrare principalmente sullo stile e sui monumenti prestigiosi oppure interessarsi di tutti i monumenti, senza distinzione di natura o statuto. L'articolo esamina alcuni di questi argomenti alla luce delle pubblicazioni recenti: in quale misura l'architettura inglese riflette il sentimento sempre più presente di un'identità nazionale? Com'erano percepiti e utilizzati i monumenti dai contemporanei? In questo modo, si chiarisce a grandi linee la dimensione coloniale del gotico inglese, secondo la sua percezione, per esempio, in Scozia e in Irlanda, giungendo alla conclusione della necessità di un approccio più europeo e meno anglo-centrico.

El estudio de la arquitectura gótica inglesa es hoy pluridisciplinar : investigadores de formaciones variadas trabajajan sobre este ámbito utilizando unos métodos y técnicas diferentes los unos de las otras. Este artículo toma en cuenta las fuerzas y los límites de la investigación reciente, subrayando la necesidad de un nuevo contexto conceptual en el cual se recobraría la escritura de la historia tradicional. Queda una incertidumbre relativa a la orientación que debe tomar la historia de la arquitectura: ¿debe concentrarse principalmente sobre el estilo y los monumentos prestigiosos o interesarse también por todos los edificios, no obstante su naturaleza y su estatuto? El artículo examina algunos de estos puntos a la luz de las recientes publicaciones: ¿cómo y por qué razones Inglaterra produjo un gótico singular? ¿En qué medida la arquitectura inglesa refleja el sentimiento cada vez más fuerte de una identidad nacional? ¿Y cómo los edificios eran percibidos y utilizados por los contemporáneos? Así se subraya a grandes rasgos la dimensión colonial del gótico inglés tal como es perceptible por ejemplo en Escocia e Irlanda, y se puede concluir sobre la necesidad de un enfoque más europeo y menos anglo-centrico.

\section{INDEX}

Keywords : medieval architecture, architecture history, British gothic, multidisciplinary approach, methodology, colonialism, gotic

Index géographique : Grande-Bretagne

Mots-clés : architecturale médiévale, gothique, histoire de l'architecture, gothique britannique, pluridisciplinarité, méthodologie, colonialisme

Index chronologique : MOYEN ÂGE

\section{AUTEURS}

\section{ROGER STALLEY}

Professeur d'histoire de l'art au Trinity College de Dublin. Il est l'auteur de plusieurs ouvrages dont Cistercian Monasteries of Ireland (1987), Ireland and Europe in the Middle Ages (1994) et Early Medieval Architecture (2000). Ses recherches portent entre autres sur l'architecture des périodes romane et sur le début du gothique, en particulier en Grande-Bretagne et en Irlande et il prépare actuellement une étude sur la sculpture irlandaise préromane. Il est membre de la Royal Irish Academy et a été élu membre de l'Academia Europæa. 